\title{
Pediatric mature B-cell non Hodgkin lymphoma treatment with LMB-96 protocol. The Children Cancer Hospital Egypt experience
}

\author{
Hany Abdel Rahman', Emad Moussa' ${ }^{2}$ Mohamed Sedky³, Iman Gouda1, \\ Madiha El Wakeel', Omneya Hassanein ${ }^{5}$ \\ ${ }^{I}$ Department of Pediatric Oncology, National Cancer Institute, Cairo University, Cairo, Egypt \\ ${ }^{2}$ Department of Pediatric Oncology, Monoufeya University, Monoufeya, Egypt \\ ${ }^{3}$ Department of Pediatrics, National Research Institute, Cairo, Egypt \\ ${ }^{4}$ Department of Pathology, National Cancer Institute, Cairo University, Cairo, Egypt \\ ${ }^{5}$ Department of Clinical research, Children Cancer Hospital Egypt, Cairo, Egypt
}

Received January 09, 2015; Revised March 17, 2015; Accepted April 01, 2015; Published Online May 06, 2015

\section{Original Article}

\begin{abstract}
Purpose: Burkitt lymphoma (BL) is a highly aggressive mature B-cell non-Hodgkin lymphoma (NHL) and is the fastest growing human tumor. The outcome of childhood NHL has improved steadily over the past decades through the use of intensive sequential multi-agent chemotherapy regimens. Methods: A retrospective study having all patients 18 years old or younger diagnosed with mature B cell NHL and treated at Children Cancer Hospital Egypt (CCHE). All children were treated according to the modified (LMB 96) protocol during the period between July 2007 and December 2012. Patients were followed up till June 2013. Results: Three hundred and seventy-seven patients were diagnosed with mature B cell NHL and received the LMB96 treatment protocol. The majorities were males $(76.4 \%)$ with a median age of 5.3 years, and ranged from $0.1-18.0$ years. The median follow-up period was 28.2 months (range 0.9-72 months). Burkitt lymphoma was the most predominant pathologic subtype $(79.6 \%, \mathrm{n}=300)$, and abdominal mass as a primary site was the most common presentation (71.3\%). Twenty seven patients (7.2\%) were treated as group A, $268(71.0 \%)$ as group B, and $82(21.8 \%)$ patients as high risk group C. Seventy-one (18.8\%) patients suffered adverse events. Major adverse events were early deaths in 17 patients (4.5\%), death during induction chemotherapy seen in 18 patients $(4.7 \%)$, and during maintenance therapy in 7 patients (1.8\%), tumor progression in 19 patients (5.0\%), and relapse in 10 patients (3.7\%). Sixty-three patients $(16.7 \%)$ died during the study period. The main causes of death were tumor lysis syndrome (TLS) in $25.3 \%$, and severe sepsis during chemotherapy in $41.3 \%$ of the patients. The 3 years OS and EFS were $83.3 \%$ and $80.4 \%$ respectively for the whole groups of patients. OS and EFS were $100 \%$ for group A, and $87.5 \% \pm 3.9 \%$ and $85.9 \pm 4.3 \%$ for group B. For group C BM ${ }^{+} / \mathrm{CNS}^{-}$patients, OS was $55.62 \% \pm 15.8 \%$, and EFS of $53.8 \% \pm 15.6 \%$. For $\mathrm{BM}^{+} / \mathrm{CNS}^{+}$ patients, OS and EFS were $63.2 \% \pm 21.76 \%$ and $57.9 \% \pm 22.1 \%$ respectively. BM-CNS ${ }^{+}$patients had OS $72.4 \% \pm 18.8 \%$ and $\mathrm{EFS}$ $67.6 \% \pm 19.7 \%$ at 36 months. Conclusion: TLS and chemotherapy related toxicity remains a major challenge affecting the outcome of pediatric mature B cell NHL. We identified bone marrow involvement as a risk factor affecting treatment outcome. Aggressive supportive care measures are mandatory to avoid unacceptable high toxicity related mortality.
\end{abstract}

Keywords: Pediatric NHL; LMB96; Children Cancer Hospital; Mature B cell Lymphoma

\section{Introduction}

Burkitt lymphoma (BL) is a highly aggressive mature B-cell non-Hodgkin lymphoma (NHL) and is the fastest growing human tumor. BL represents $40 \%$ of all childhood NHL and $3-4 \%$ of all childhood malignancies diagnosed each year in the USA. ${ }^{1,2}$ Its annual incidence in Africa has been estimated at $40-50$ per million children younger than 18 years compared to 8 cases per million in France, and 7 per million in The Netherlands. ${ }^{3}$ The outcome of childhood NHL has improved steadily over the past decades through the use of intensive sequential multi-agent chemotherapy regimens. In high-income countries, 5-year survival rates reaches $90 \%$ in patients treated according to the LMB 96 or BFM protocols 4-8, while the therapy offered in oncology units in low-income countries is not as aggressive, and outcome is not as good. ${ }^{9}$ The aim of the current study is to report the treatment outcome, overall survival (OS) and event free survival (EFS) of patients who received FAB LMB96 protocol at the Pediatric Oncology Department, Children Cancer Hospital- Egypt (CCHE) during a 5.5 years period. Also to report about incidence of tumor lysis syndrome, relapse rate, treatment related mortality and causes of death in these patients.

Corresponding author: Hany Abdel Rahman; Department of Pediatric Oncology, National Cancer Institute, Cairo University, Egypt. 


\section{Methods and Materials}

A retrospective study having all pediatric patients diagnosed with mature B cell NHL and treated at CCHE. All children were treated according to the modified (LMB 96) protocol during the period between July 2007 and December 2012, and were followed up till June 2013. Approval by our institutional scientific committee and informed written consent were obtained prior to starting chemotherapy.

\section{Eligibility and risk stratification}

Newly diagnosed children and adolescents ( $<18$ years) with mature B-cell NHL were included in our study. Diagnosis was done according to the WHO classification and included Burkitt lymphoma (BL), Burkitt-like lymphoma (BLL), Diffuse large B cell lymphoma (DLBCL), mediastinal large B-cell lymphoma (MLBCL), and mature B-cell neoplasm not otherwise specified (NOS). ${ }^{10}$ Staging was performed according to Murphy's classification. ${ }^{11}$

Risk classification according to LMB96 protocol was defined as low risk group A with resected stage I and abdominal completely resected stage II; high risk group $\mathrm{C}$ with bone marrow disease (25\% L3 blasts) or CNS disease defined by one or more of the following: any L3 CSF blast, cranial nerve palsy, clinical spinal cord compression, isolated intracerebral mass, cranial or spinal parameningeal extension; and intermediate risk group $B$, included all patients not eligible for group A or C. Exclusions to study enrollment included severe immunodeficiency syndromes, HIV positivity, previous malignancy, or prior chemotherapy.

\section{Treatment}

Chemotherapy was given according to the FAB LMB96 protocol

\section{Group A}

Patients assigned to group A received two courses of cyclophosphamide, vincristine, prednisone, and doxorubicin (COPAD) without intrathecal (IT) chemotherapy. ${ }^{8}$

\section{Group B}

Patients received prophase cyclophosphamide, vincristine, and prednisone (COP), followed by induction chemotherapy consisting of two cycles of vincristine, prednisone, adriamycin, methotrexate, cyclophosphamide and intrathecal injection (COPADM), and two courses of cytarabine, methotrexate (CYM) as consolidation if they were in complete remission (CR) post first course of consolidation Patients with less than a $20 \%$ response on day 7 of COP and patients with residual disease after CYM-1 were upgraded to group C starting from CYVE1. ${ }^{12}$

\section{Group C}

Following prophase COP, patients had induction chemotherapy which consisted of two cycles of COPADM
(HD-MTX $8 \mathrm{~g} / \mathrm{m}^{2}$ ) followed by consolidation with 2 cycles of high-dose continuous infusion of cytarabine plus etoposide (CYVE). Patients with CNS disease received additional IT therapy as well as an additional HD-MTX course between consolidation courses. At last, 4 maintenance cycles, the first consisted of COPADM, followed by three cycles with low dose cytarabine and etoposide (cycles 2 and 4), with the third cycle being similar to the first but without HDMTX and IT. ${ }^{13}$

\section{Criteria of response}

Complete response (CR) was defined as complete disappearance of all tumor masses, partial response (PR; 20\% - 99\% tumor reduction), no response (NR) or stable disease (SD); $<20 \%$ tumor reduction. Progressive disease (PD) was $>25 \%$ increase in tumor size, while relapse was defined as recurrence of disease at any site after achieving CR. Failure was considered as relapses, deaths and failures to achieve a CR within the time frame.

Unresponsiveness to initial COP in itself was not considered a failure of the treatment strategy. Patients in critical condition (renal failure, sepsis, grade III/IV organ toxicity) were allowed to receive a second course of COP prior to proceeding to induction. ${ }^{13}$

\section{Statistical methods}

Data was analyzed using the Statistical Package for Social Sciences (SPSS) for Windows package version 15 (SPSS Inc., Chicago, Illinois, USA). Numerical data was presented as mean \pm standard deviation (SD), median and range. Qualitative data was presented as numbers and percentages. Kaplan Meier was used to estimate survival and Log rank test for comparison. Overall survival (OS) was defined as the time from diagnosis till the end of the study period or death, while EFS was defined as the minimum period from diagnosis till the occurrence of an event including induction failure, disease progression, relapse, second malignancy, lost FU or death from any cause. A $P$-value $\leq 0.05$ was considered significant.

\section{Results}

Patients' characteristics: out of the 530 patients diagnosed with NHL between July 2007 and December 2012, 377 (71.2\%) patients had mature B cell NHL and received the LMB96 treatment protocol. There was a significant male predominance with 288 male patients (76.4\%) and $89 \mathrm{Fe}-$ males $(23.6 \%)$. The median age was 5.3 years, and ranged from 0.1-18.0 years. Number of patients varied in age group, with the range from 0 to 4 years being the most common (44.0\%).

Demographic characteristics of study patients are summarized in Table 1, while Table 2 shows common sites of CNS involvement. 
TABLE 1: Initial characteristics of the 377 studied patients.

\begin{tabular}{|c|c|c|}
\hline & Frequency & Percent \\
\hline \multicolumn{3}{|l|}{ Age } \\
\hline $0-4$ years & 166 & 44.0 \\
\hline $5-9$ & 127 & 33.7 \\
\hline $10-14$ & 62 & 16.4 \\
\hline $15-19$ & 22 & 5.8 \\
\hline \multicolumn{3}{|l|}{ Sex } \\
\hline Male & 288 & 76.4 \\
\hline Female & 89 & 23.6 \\
\hline \multicolumn{3}{|l|}{ Histology } \\
\hline Burkitt lymphoma (BL) & 300 & 79.6 \\
\hline B-cell (L3) leukemia & 54 & 14.3 \\
\hline DLBC & 18 & 4.8 \\
\hline MLBCL & 3 & 0.8 \\
\hline Mature B-NHL NOS & 2 & 0.5 \\
\hline \multicolumn{3}{|l|}{ Primary site } \\
\hline Abdomen & 269 & 71.3 \\
\hline Peripheral lymph nodes & 121 & 32.0 \\
\hline Thorax (not primary MDLBC) & 87 & 23.0 \\
\hline Head and neck & 62 & 16.4 \\
\hline Other tumor site & 12 & 3.1 \\
\hline \multicolumn{3}{|l|}{ Modified Murphy's staging } \\
\hline Stage I & 18 & 4.8 \\
\hline Stage II & 110 & 29.2 \\
\hline Stage III & 167 & 44.3 \\
\hline Stage IV & 28 & 7.4 \\
\hline ALL L3 & 54 & 14.3 \\
\hline \multicolumn{3}{|l|}{ Clinical Group } \\
\hline Group A & 27 & 7.2 \\
\hline Group B & 268 & 71.0 \\
\hline Group C & 82 & 21.8 \\
\hline \multicolumn{3}{|l|}{$\mathrm{BM} / \mathrm{CNS}$ involvement } \\
\hline $\mathrm{BM}^{+} / \mathrm{CNS}^{-}$ & 41 & 50 \\
\hline $\mathrm{BM}^{-} / \mathrm{CNS}^{+}$ & 22 & 26.8 \\
\hline $\mathrm{BM}^{+} / \mathrm{CNS}^{+}$ & 19 & 23.2 \\
\hline
\end{tabular}

$\mathrm{BM}=$ Bone marrow; DLBC= Diffuse large B-cell Lymphoma; MLBCL= Mediastinal large B-cell lymphoma; NOS= Non Otherwise specified; $\mathrm{CNS}=$ Central nervous system.

TABLE 2: Common sites of CNS involvement.

\begin{tabular}{lcc}
\hline \hline & Total number & Percentage \\
\hline CNS involvement & 41 & 10.9 \\
Parameningeal extension & 26 & 6.9 \\
Blasts in CSF & 9 & 2.4 \\
Clinically manifest spinal & 6 & 1.6 \\
cord compression & & \\
Cranial nerve palsy & 5 & 1.3 \\
Intra-orbital extension & 1 & 0.3 \\
\hline \hline
\end{tabular}

$\mathrm{CSF}=$ Cerebrospinal fluid.

Burkitt lymphoma was the most predominant pathologic subtype $(79.6 \%, \mathrm{n}=300)$, while abdominal mass as a primary site was the most common presentation $(71.3 \%, \mathrm{n}=269)$. According to the modified Murphy Staging, stage III was the most common presentation seen in 167 patients (44.8\%) Table 1.

\section{Tumor Lysis Syndrome (TLS)}

Laboratory and/or clinical tumor lysis syndrome was observed in 57 patients (15.1\%); of which 30 (11.2\%) out of 268 patients were group B, and 27(32.9\%) out of 82 in group C. Sixteen patients $(4.2 \%)$ died out of TLS during the prophase chemotherapy (COP). They represent most of the mortality causes during this period of chemotherapy $(16 / 17=94.1 \%)$. Table 3 shows the major adverse events in studied patients.

\section{Chemotherapy outcome}

Following the LMB96 protocol risk stratification, 27 patients $(7.2 \%)$ treated as group A, 268 patients $(71.0 \%)$ as group B, and 82 patients $(21.8 \%)$ in high risk group $\mathrm{C}$.

\section{Group A}

All 27 patients stratified as group A (7.2\%) received chemotherapy successfully, and are alive in CR.

\section{Group B}

Two hundred-sixty eight patients were stratified as group B. Following initial COP course of chemotherapy; 218 (81.3\%) had $>20 \%$ radiologic response and continued on same line. Twelve patients $(3.18 \%)$ died from severe sepsis and/or TLS during prophase. Twenty eight patients (10.4\%) didn't receive COP and started COPAM directly as they were considered to have no bulky tumor (stage II unresected tumor and/or incomplete resection anastomosis of primary intestinal mass). Ten patients (3.7\%) had mild to no response defined as less than $20 \%$ decrease in the initial tumor volume and were upgraded to group C. Eight patients (2.9\%) died from sever sepsis during chemotherapy, 12 (4.4\%) progression, and 8 (2.9\%) relapses (Table 3 ).

\section{Group C}

Eighty two patients were treated as group C. They were 41

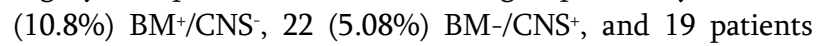
(5.0\%) $\mathrm{BM}^{+} / \mathrm{CNS}^{+}$. Twenty two patients $(5.8 \%)$ had treatment related mortality, while 7 patients $(1.8 \%)$ had tumor progression, and 2 patients $(0.5 \%)$ relapsed.

\section{Events, relapses and tumor progression}

Seventy-one patients (18.8\%) suffered adverse events, 40 $(10.6 \%)$ in group B, and $31(8.2 \%)$ in group C. There were no events in group A patients. Major adverse events were early deaths in 17 patients $(4.5 \%)$, death during induction chemotherapy seen in 18 patients $(4.7 \%)$, death during maintenance therapy in 7 patients (1.8\%), tumor progression in 19 patients $(5.0 \%)$, and relapse in 10 patients (3.7\%). Table 3 describes major events according to risk groups.

\section{Survival}

By the end of our study, 291 patients were alive in CR (77.2\%) while 63 patients $(16.7 \%)$ died. Twenty patients (5.3\%) lost FU (5 patients; $1.3 \%$ in active disease, and 15 patients; $4.0 \%$ in CR) as shown in Table 4. 
TABLE 3: Adverse Events in patients with NHL.

\begin{tabular}{|c|c|c|c|c|c|c|}
\hline & \multicolumn{2}{|c|}{ Death } & \multirow[b]{2}{*}{ Consolidation } & \multirow[t]{2}{*}{ Progression } & \multirow[t]{2}{*}{ Relapse } & \multirow[t]{2}{*}{ Total } \\
\hline & Prophase & Induction & & & & \\
\hline Group B & 12 & 6 & 2 & 12 & 8 & 40 \\
\hline Group C & 5 & 12 & 5 & 7 & 2 & 31 \\
\hline $\mathrm{BM}^{+} / \mathrm{CNS}^{-}$ & 3 & 5 & 2 & 3 & 1 & 14 \\
\hline $\mathrm{BM}^{-} / \mathrm{CNS}^{+}$ & 1 & 2 & 1 & 1 & 1 & 6 \\
\hline $\mathrm{BM}^{+} / \mathrm{CNS}^{+}$ & 1 & 5 & 2 & 3 & 0 & 11 \\
\hline Total & 17 & 18 & 7 & 19 & 10 & 71 \\
\hline
\end{tabular}

TABLE 4: Disease Status in the studied patients.

\begin{tabular}{lcc}
\hline \multicolumn{3}{c}{ Disease Status } \\
\hline & Frequency & Percent \\
\cline { 2 - 3 } Alive in Active Disease & 3 & 0.8 \\
Alive in CR & 291 & 77.2 \\
Died in Active Disease & 57 & 15.1 \\
Died in CR & 6 & 1.6 \\
Lost Follow Up in Active Disease & 5 & 1.3 \\
Lost Follow Up in CR & 15 & 4.0 \\
\hline \hline
\end{tabular}

The 3 years OS and EFS were 83.3\% and 80.4\% respectively for the whole group of patients Figures 1 and 2.



FIG. 1: Overall survival for all Patients (83.3\%).

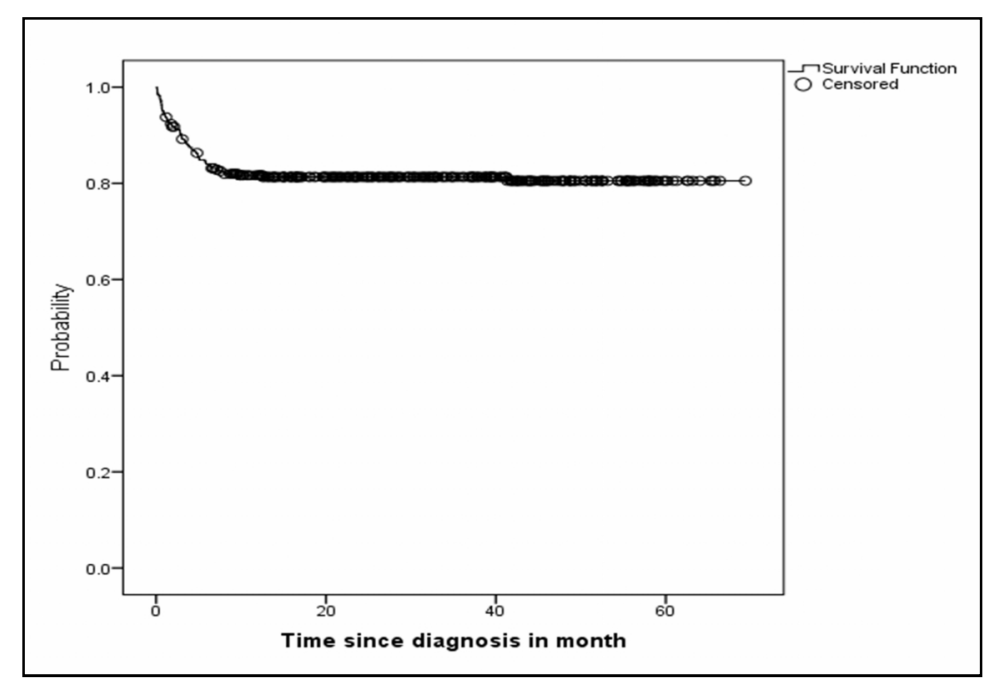

FIG. 2: Event-free survival for all patients (80.4\%). 


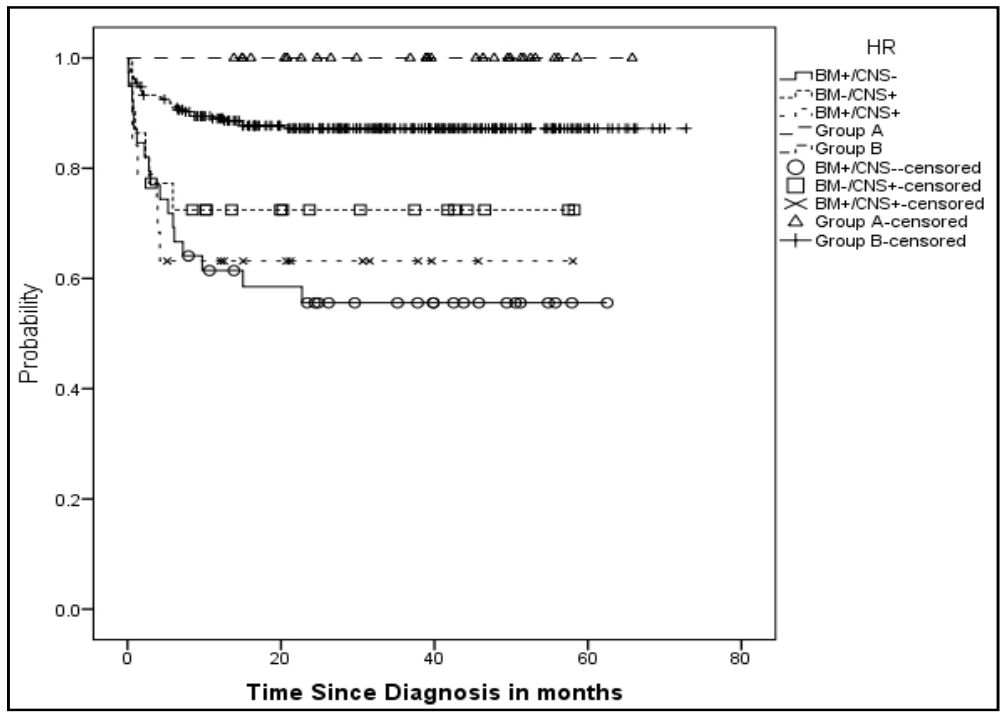

FIG. 3: Overall survival in different group categories.

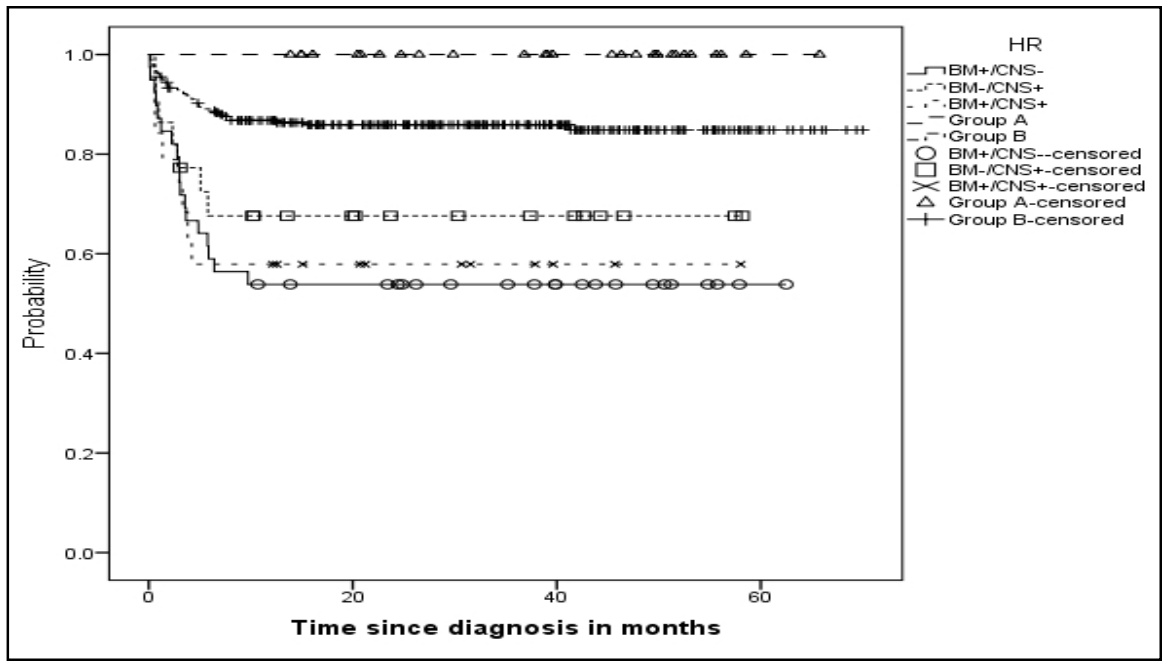

FIG. 4: Event free survival in different group categories.

OS and EFS were $100 \%$ for group A, and $87.5 \% \pm 3.9 \%$ and $85.9 \pm 4.3 \%$ for group B. For group $\mathrm{C} \mathrm{BM}^{+} / \mathrm{CNS}^{-}$patients, OS was $55.62 \% \pm 15.8 \%$, and EFS of $53.8 \% \pm 15.6 \%$. For $\mathrm{BM}^{+} / \mathrm{CNS}^{+}$ patients, OS and EFS were $63.2 \% \pm 21.76 \%$ and $57.9 \% \pm 22.1 \%$ respectively. $\mathrm{BM}^{-} / \mathrm{CNS}^{+}$patients had OS $72.4 \% \pm 18.8 \%$ and EFS $67.6 \% \pm 19.7 \%$ at 36 months (Figure 3 and 4 ).

The mean FU period was 29.2 months (range 0.9 - 72 months), with an SD 19.2 months. Median FU period of all patients was 28.2 months for all patients.

\section{Discussion}

This retrospective study describes the treatment outcome of pediatric patients treated on the LMB-96 in our institute during 5.5 years period. To the best of our knowledge, it's one of the largest single center patient's series in the Middle East region. Mean age of the patients is slightly lower than many previous studies $5,12,14$, but similar to our previous re- port ${ }^{15}$. Age category below 4 years was the most common in the studied group in contrast to other study groups ${ }^{13,16}$, were ages between 5-9 years being the most common, although age is not considered as risk factor ${ }^{17}$. Cairo et al. in 2012 identified advanced stage, increased $\mathrm{LDH}$ level $\geq 2 \mathrm{X}$ normal value, mediastinal disease and combined $\mathrm{BM}^{+} / \mathrm{CNS}^{+}$as the main risk factors for treatment response. ${ }^{17}$ In our study, analysis of prognostic factors including age, gender, upfront tumor resection and CNS status were all statistically insignificance. Nevertheless, BM involvement was found to be associated with lower OS and EFS, but this difference was statistically insignificant probably due to small sample size.

The obvious male predominance observed in the current study is a common finding in pediatric mature B cell $\mathrm{NH}^{5,12}$, 13, 15-20. BL and B-ALL were the predominant pathologic subtype followed by DLBC of the studied patients, but in different ratio, as they represented $5 \%$ of the patients compared to $14 \%{ }^{12}, 10 \%{ }^{13}, 11.7 \%{ }^{15}, 14.9 \%{ }^{14}$ and $31.4 \%{ }^{16}$ of 
the patients in different studies. Other rare subtypes observed were PMLBCL and mature B-NHL NOS.

In most studies reporting pediatric mature B cell NHL -including the present one- abdominal mass, stage III, group $\mathrm{B}$ were the most common presentation, followed by peripheral lymph enlargement including head and neck regions, mediastinal mass, CNS involvement but with different incidences according to the studies. ${ }^{5,12,14,15-17,20}$

In the current study, $15.1 \%$ of the patients had laboratory and/or clinical TLS, representing $11.1 \%$ of group B and $32.9 \%$ of group $\mathrm{C}$ patients reflecting its increased incidence in close association with tumor aggressiveness. Management of TLS was done using the administration aggressive hydration and allopurinol as non-recombinant urate oxidase is not available routinely in our country. In previous, studies laboratory TLS incidence ranged from $27 \%$ to $42 \%$ of the patients ${ }^{21-24}$, although clinical TLS incidence is much lower ranging from $4.4 \%$ to $8.4 \%{ }^{21-23}$.

Stage I disease represented $4.8 \%$, stage II $29.1 \%$, stage III $44.8 \%$, stage IV $21.7 \%$, while L3 ALL were $14.3 \%$. Similarly, few authors reported the same incidence in stage $I^{5,15,20}$, and stage III disease $5,14,17,20$. Our results are higher in stage II compared to few reports. ${ }^{5,15,17}$ On the other hand, we report lower incidence in stage IV disease 5, 15-17, 20and L3 ALL than some studies ${ }^{5,16}$.

Low risk group A patients represented 7.2\% compared to $71.0 \%$ group B and $21.8 \%$ for high risk group C patients. In group A patients, incidence is in concordance with Patte et $a l^{5}$, and higher than our previous report ${ }^{15}$, this is probably be due to accumulation of experience and better risk stratification. In group B and C patients, similar incidence was reported in the literature. ${ }^{5,15,17}$ Reiter et al. reported different incidence: $17 \%$ in $\mathrm{R} 1,40 \%$ in $\mathrm{R} 2$ and $43 \%$ in $\mathrm{R} 3$ group of patients but with a risk stratification based on tumor resection, LDH level, and localization of the primary tumor. ${ }^{14}$ In our study, LDH was not considered as it was not part of the risk stratification.

Following initial cytoreductive chemotherapy, 90.8\% of the patients were good responders and continued as group B, while $3.7 \%$ were upgraded to group C. Patte et al. reported $95 \%$ response to prophase treatment ${ }^{5,12}$ and $4.9 \%{ }^{12}, 4.5 \%{ }^{5}$ as non-responders. We previously reported a similar response rate. ${ }^{15}$ Ten percent of the patients underwent incomplete resection of their abdominal mass, or had small initial tumor size, hence we estimated no need for cytoreductive course of chemotherapy and they received COPADM1 directly.

We report a high mortality rate $(16.7 \%)$ in our study when compared to $7.3 \%$ reported in FAB LMB 89 study ${ }^{5}, 3.3 \%$ in NHL-BFM 90 study ${ }^{15}, 4.6 \%$ in LMB 96 high risk patients ${ }^{13}$.
Another large-scale study resulted in a cure rate above $90 \%$ with a $7.7 \%$ mortality rate, and $<1 \%$ toxic death in childhood B-NHL. ${ }^{16}$

Analyzing this high mortality rate, out of $16.7 \%$ died during therapy; $4.2 \%$ died directly or indirectly due to TLS, $6.9 \%$ out septicemia during chemotherapy, and 5.3\% due to tumor progression. Again, this very high induction mortality rate was due to sepsis following grade IV neutropenia. Reiter et al. reported $3.3 \%$ deaths from different causes including acute TLS, toxic death or infection, but none during the course of chemotherapy ${ }^{14}$, while Patte et al. reported $7.3 \%{ }^{12}$.

Deaths during induction period (28\%) were mostly following COPADM1 course, probably due to accumulated hematologic toxicity of the two courses given in short duration, while high dose Ara-C and related toxicity was the main cause of death during consolidation $(11 \%)$ in group $\mathrm{C}$ patients. Similarly, life threatening infections were reported during COPADM1 and CYVE 5, 25. Other causes of death were tumor progression in $10 \%$ of the patients.

Our 3-years OS and EFS were $83.3 \%$ and $80.4 \%$ respectively. The current results are similar to what we have reported previously ${ }^{15}$, but are worse than most of the studies using similar protocols or multi-drug combination. Patte et al. reported 5 years OS $92.5 \%$ and EFS 91\% in 2001. ${ }^{5}$ Six years EFS $89 \% \pm 2 \%$ was reported by Reiter et al. ${ }^{14}$, while 3 years EFS was $88 \% \pm 1 \%$ was reported by Cairo et al. ${ }^{17} \mathrm{~A}$ recent Japanese study reported 4 years OS and EFS were $92.7 \%$ and $87.4 \%$ respectively ${ }^{16}$. For group A patients, we confirm our excellent results obtained previously. ${ }^{15}$ The same is reported by most of the study groups that resected stage I and abdominal stage II have excellent prognosis regardless of the chemotherapy regimen given $5,12,14,16$. Four years EFS reported by Gerrad et al. was $98.3 \%$, while OS was $99.2 \% .^{8}$ OS and EFS for low risk patients were between $98 \%$ to $100 \%$ in most of the studies. ${ }^{5,14-17}$

For group B patients, OS and EFS were $87.5 \% \pm 4.1 \%$ and $85.9 \% \pm 4.3 \%$ respectively, lower than most studies performed as $92 \%{ }^{5,12}, 96 \%{ }^{14}, 89 \%{ }^{17}$, and $93.6 \%{ }^{16}$. This could be explained by high rate of chemotherapy related mortality $(11.1 \%)$, as relapse rate and disease progression in our study $(8.7 \%)$ were not higher than any study group report. ${ }^{12,14,16}$

Fifty percent of group C patients in our study were $\mathrm{BM}^{+}$, while $26 \%$ had CNS involvement and $24 \%$ were both $\mathrm{BM}^{+}$ and $\mathrm{CNS}^{+}$. Similar incidence rate was reported. ., 13-16 $^{-16}$

Our worst results are in high risk group $\mathrm{C} \mathrm{BM}^{+} / \mathrm{CNS}^{-}$, and $\mathrm{BM}^{+} / \mathrm{CNS}^{+}$patients. OS was $55.62 \% \pm 15.8 \%$ and $63.2 \% \pm 21.76$ $\%$ respectively, while EFS was $53.8 \% \pm 15.6 \%$ and $57.9 \% \pm$ $22.1 \%$ respectively. The sub-group of patients with BM involvement at diagnosis was associated with poor outcome, compared to CNS involvement. To the contrary of most of 
the studies, combined $\mathrm{BM}^{+}$and $\mathrm{CNS}^{+}$, or those with $\mathrm{CNS}^{+}$ disease had the worst outcome $5,13,16,17,26-29$ and B-ALL had excellent overall survival; $88 \% 5,13,14,27,28$, and $86.2 \% \pm 4.0 \%$ 16. This might be due to the fact that patients with BM involvement express more hematologic toxicity and therapy related mortality.

Patte et al. identified CNS involvement as the only prognostic factor in group C patients. ${ }^{5}$ Similar conclusion was reported by most of the study groups. ${ }^{13,14,16,17,25}$

\section{Conclusion}

In conclusion, chemotherapy related toxicity remains a major challenge affecting the outcome of pediatric mature B cell NHL. We identified BM involvement, and treatment related toxicity during prophase/induction period of treatment as risk factors affecting outcome. Aggressive supportive care measures are mandatory to avoid unacceptable high toxicity related mortality.

\section{Conflict of interest}

The authors declare that they have no conflicts of interest. The authors alone are responsible for the content and writing of the paper.

\section{References}

1. Sandlund JT, Downing JR, Crist WM. Non-Hodgkin's lymphoma in childhood. $N$ EnglJ Med 1996; 334:1238-48.

2. Cairo MS, Perkins SL. Non-Hodgkin's lymphoma in children. In: Cancer Medicine (ed. by RC Bast, Jr, DW Kufe, RE Pollock, RR Weischselbaum, JF Holland \& E. Frei, III), 5 ${ }^{\text {th }}$ edn, 2162-7. B.C. Decker Inc, London; 2000.

3. Orem J, Mbidde EK, Lambert B, et al. Burkitt's lymphoma in Africa, a review of the epidemiology and etiology. Afr Health Sci 2007; 7:166-75.

4. Reiter A. Diagnosis and treatment of childhood non-hodgkin lymphoma. Hematology Am Soc Hematol Educ Program 2007:285-96.

5. Patte C, Auperin A, Michon J, et al. The Société Française d'Oncologie Pédiatrique LMB89 protocol: highly effective multiagent chemotherapy tailored to the tumor burden and initial response in 561 unselected children with B-cell lymphomas and L3 leukemia. Blood 2001; 97:3370-9.

6. Woessmann W, Seidemann K, Mann G, et al. The impact of the methotrexate administration schedule and dose in the treatment of children and adolescents with B-cell neoplasms: a report of the BFM Group Study NHL-BFM95. Blood 2005; 105:948-58.
7. Patte C. Treatment of mature B-ALL and high grade B-NHL in children. Best Pract Res Clin Haematol 2002; 15:695-711.

8. Gerrard M, Cairo MS, Weston C, et al. Excellent survival following two courses of COPAD chemotherapy in children and adolescents with resected localized B-cell non-Hodgkin's lymphoma: results of the FAB/LMB 96 international study. Br J Haematol 2008; 141:840-7.

9. Molyneux EM, Rochford R, Griffin B, et al. Burkitt's lymphoma. Lancet 2012; 379:1234-44.

10. Jaffe ES, Harris NL, Stein H, et al., editors. WHO classification of tumors, pathology and genetics of tumors of haematopoietic and lymphoid tissues. Lyon: IARC Press; 2001.

11. Murphy SB. Classification, staging and end results of treatment of childhood non-Hodgkin's lymphomas: dissimilarities from lymphomas in adults. Semin Oncol1980; 7:332-9.

12. Patte C, Auperin A, Gerrard M, et al. Results of the randomized international FAB/LMB96 trial for intermediate risk B-cell non-Hodgkin lymphoma in children and adolescents: It is possible to reduce treatment for the early responding patients. Blood 2007; 109: 2773-80.

13. Cairo MS, Gerrard M, Sposto R, et al. Results of a randomized international study of high-risk central nervous system B non-Hodgkin lymphoma and B acute lymphoblastic leukemia in children and adolescents. Blood 2007; 109:2736-43.

14. Reiter A, Schrappe M, Tiemann M, et al. Improved treatment results in childhood B-cell neoplasms with tailored intensification of therapy: A report of the Berlin-Frankfurt-Münster Group Trial NHL-BFM 90. Blood 1999; 94:3294-306.

15. Abd El-Rahman H, Bedair RM. Treatment Outcome of Pediatric Patients with Mature B Cell Lymphoma Receiving Fab LMB96 Protocol at the National Cancer Institute, Cairo University. J Egypt Natl Canc Inst 2010; 22:201-8.

16. Tsurusawa M, Mori T, Kikuchi A, et al. Improved treatment results of children with B-cell non-Hodgkin lymphoma: a report from the Japanese Pediatric Leukemia/Lymphoma Study Group B-NHL03 study. Pediatr Blood Cancer 2014; 61:1215-21.

17. Cairo MS, Sposto R, Gerrard M, et al. Advanced stage, increased lactate dehydrogenase, and primary site, but not adolescent age ( $\geq 15$ years), are associated with an increased risk of treatment failure in children and adolescents with mature B-cell non-Hodgkin's lymphoma: results of the FAB LMB 96 study. JClin Oncol 2012; 30:387-93.

18. Patte C. B-acute lymphoblastic leukemia: The European experience. Int J Pediatr Hematol Oncol 1998; 5: 81-8. 
19. National Cancer Institute sponsored study of classifications of non-Hodgkin's lymphomas: summary and description of a working formulation for clinical usage. The Non-Hodgkin's Lymphoma Pathologic Classification Project. Cancer 1982; 49:2112-35.

20. El Shazly M, Arafaat W, El Masry A, et al. Non Hodgkin Lymphoma In Children From Northern Egypt. Blood 2013; 122: 5062.

21. Hande KR, Garrow GC. Acute tumor lysis syndrome in patients with high-grade non-Hodgkin's lymphoma. Am J Med 1993; 94:133-9.

22. Cairo MS, Bishop M. Tumour lysis syndrome: new therapeutic strategies and classification. $\mathrm{BrJHae}-$ matol 2004; 127: 3-11.

23. Wossmann W, Schrappe M, Meyer U, et al. Incidence of tumor lysis syndrome in children with advanced stage Burkitt's lymphoma/leukemia before and after introduction of prophylactic use of urate oxidase. Ann Hematol 2003; 82:160-5.

24. Banks PM, Arseneau JC, Gralnick HR, et al. American Burkitt's lymphoma: a clinicopathologic study of 30 cases. II. Pathologic correlations. Am JMed $1975 ; 58: 322-9$.
25. Galicier L, Fieschi C, Borie R, et al. Intensive chemotherapy regimen (LMB86) for St Jude stage IV AIDS-related Burkitt lymphoma/leukemia: a prospective study. Blood 2007; 110: 2846-54.

26. Spreafico F, Massimino M, Luksch $\mathrm{R}$, et al. Intensive, very short-term chemotherapy for advanced Burkitt's lymphoma in children. JClin Oncol 2002; 20:2783-8.

27. Thomas DA, Cortes J, O'Brien S, et al. Hyper-CVAD program in Burkitt's-type adult acute lymphoblastic leukemia. J Clin Oncol 1999; 17:2461-70.

28. Mead GM, Sydes MR, Walewski J, et al. An international evaluation of CODOX-M and CODOX-M alternating with IVAC in adult Burkitt's lymphoma: results of United Kingdom Lymphoma Group LY06 study. Ann Oncol 2002; 13:1264-74.

29. Patte C, Philip T, Rodary C, et al. Improved survival rate in children with stage III and IV B cell non-Hodgkin's lymphoma and leukemia using multi-agent chemotherapy: results of a study of 114 children from the French Pediatric Oncology Society. JClin Oncol 1986; 4:1219-26. 